\title{
The role of preoperative 3D-ultrasound in intraoperative judgement for hysteroscopic adhesiolysis
}

\author{
Arvind Burjoo ${ }^{1 \#}$, Xingping Zhao ${ }^{1 \#}$, Lingxiao Zou ${ }^{1}$, Xinyi Liu ${ }^{1}$, Lei Lei ${ }^{1}$, Baiyun Zhang ${ }^{2}$, Dabao Xu ${ }^{1}$ \\ ${ }^{1}$ Department of Obstetrics and Gynecology, Third Xiangya Hospital of Central South University, Changsha 410013, China; ${ }^{2}$ Department of \\ Ultrasound, Hunan Guangxiu Hospital, Changsha 410013, China \\ Contributions: (I) Conception and design: D Xu, A Burjoo; (II) Administrative support: D Xu; (III) Provision of study materials or patients: D Xu, \\ B Zhang; (IV) Collection and assembly of data: X Zhao, X Liu; (V) Data analysis and interpretation: X Zhao, L Zou; (VI) Manuscript writing: All \\ authors; (VII) Final approval of manuscript: All authors. \\ "These authors contributed equally to this work as co-first authors. \\ Correspondence to: Dabao Xu, MD. Department of Obstetrics and Gynecology, Third Xiangya Hospital of Central South University, 138 Tongzipo \\ Rd., Changsha 410013, China. Email: dabaoxu@yahoo.com; Baiyun Zhang, MD. Department of Ultrasound, Hunan Guangxiu Hospital, No. 8, \\ Luyun Road, Changsha 410013, China. Email: 158478968@qq.com.
}

Background: Hysteroscopic adhesiolysis (HA) remains the mainstay of treatment for intrauterine adhesions (IUA). In cases of moderate or severe IUA, the assistance of various adjunctive aids are usually sought to improve HA's success rate. Among these, intraoperative transabdominal ultrasound (TAS) is the most common; however, it has certain limitations. Preoperative three-dimensional transvaginal ultrasound (3D-TVUS) has been accepted as a non-invasive way to provide accurate information about the uterine cavity. This prospective, non-randomized controlled study will assess the effects of pre-operative 3D-TVUS prior to HA in improving the surgeon's intraoperative judgement.

Methods: A total of 362 patients, who met the inclusion criteria, aged between 18 and 45 years and diagnosed with moderate or severe IUA underwent HA at our hospital from March 2018 to December 2018. Participants were divided into 2 groups; the study group; $n=182$ performed 3D-TVUS evaluation prior to HA, and the control group; $n=180$ underwent HA without preoperative 3D-TVUS evaluation. The following basic information were collected prospectively for both groups: age, parity, history of abortion, degree of IUA, surgical complications and number of hysteroscopic interventions. The data obtained from 3D-TVUS in the study group was carefully studied at the preoperative stage by the operator and was integrated into intraoperative findings, further assisting with intraoperative decisions. The guiding value of preoperative 3D-TVUS for HA was evaluated by comparing and analyzing the postoperative exposure rate of clearly visible tubal ostia between the groups.

Results: Based on the basic information $(\mathrm{P}>0.05)$ collected preoperatively, there were no statistically significant differences between the groups. Postoperatively, the study group had a better surgical success rate with a more significant AFS score reduction $(4.71 \pm 2.05 ; \mathrm{P}<0.0001)$ and better morphological restoration of the uterine cavity, with more adhesion-free uterine horns and more clearly visible fallopian tube ostia $(\mathrm{P}<0.0001)$ following HA.

Conclusions: This study showed that preoperative 3D-TVUS evaluation helped the hysteroscopists with their intraoperative decision-making while carrying out HA. In comparison to those who did not perform preoperative 3D ultrasound, those who underwent 3D-TVUS evaluation had a better surgical success rate in retrieving the fallopian tube ostia and the restoration of normal uterine cavity morphology.

Keywords: Preoperative three-dimensional transvaginal ultrasound (preoperative 3D-TVUS); intrauterine adhesions (IUA); intraoperative judgement; hysteroscopic adhesiolysis (HA); surgical success rate 
Submitted Nov 29, 2019. Accepted for publication Dec 30, 2019.

doi: $10.21037 /$ atm.2020.01.06

View this article at: http://dx.doi.org/10.21037/atm.2020.01.06

\section{Introduction}

Intrauterine adhesions (IUA) refer to partial or complete adhesions which occur between the uterine walls and may result in several clinical manifestations such as hypomenorrhea, amenorrhea, dysmenorrhea, low abdominal pain, sub-fertility/infertility, recurrent abortions, premature delivery, and abnormal placental implantation. IUA can be either primary, after pregnancyrelated curettage or hysteroscopic surgery, or secondary, re-occurring after adhesiolysis has been performed $(1,2)$. Dilation and curettage (D \& C) after miscarriage accounts for $93 \%$ of IUA (3). IUA are significant but usually (partly) correctable cause of infertility. When they are treated, fertility outcomes can be improved and symptoms can be relieved or resolved. Accurate identification of the extent and character of adhesions and reliable diagnostic tools for assessment of the uterine cavity is a necessary first step in improving adhesiolysis success rates. Several diagnostic modalities have been proposed for the diagnosis of IUA: hysterosalpingography (HSG), saline infusion/ contrast sonohysterography (SHG), 3-D ultrasonography, diagnostic hysteroscopy and magnetic resonance imaging (MRI). Diagnostic hysteroscopy is considered the gold standard among these studies as it more accurately confirms the presence, extent, and morphological characteristics of adhesions, as well as the quality of the endometrium, and helps in classification and concurrent treatment of IUA (4). However, there are disadvantages with diagnostic hysteroscopy, the most important of which is its inability to access and assess the intrauterine cavity in cases of severe cervical stenosis and severe IUA. This is a particular problem with lower segment obliteration, when the hysteroscope is unable to reach the cavity beyond the point of obliteration. Moreover, it is associated with complications such as cervical laceration, uterine perforation, bleeding, reactions to the distention media, and anesthesia. Surgery is considered as the main therapy for IUA, with no role for medical management. Lysis of IUA under direct hysteroscopic visualization is considered as the treatment of choice for IUA (4). HA aims to restore a normal uterine cavity, prevent recurrence of adhesions, normalize menstrual flow and improve reproductive outcome. Filmy adhesions (especially central cavity lesions) can be bluntly lysed with cavity distension and by the tip of the hysteroscope or blunt dissecting forceps. In cases of severe adhesions where various segments are inaccessible or at least one of the ostia cannot be seen or retrieved, or in cases of cervical stenosis, however, HA proves to be very challenging, and can result in difficulties with intraoperative judgement and surgical complications. When the severity of the adhesion hinders the procedure, the assistance of TAS is usually sought. In cases of severe IUA, intraoperative ultrasound monitoring is very important for HA; however, the timing for ultrasound is not ideal in the proliferative phase, as the endometrium is very thin during this particular period. Furthermore, the imaging quality of portable TAS is usually not very appreciable and is unable to provide precise information intraoperatively, unlike preoperative threedimensional transvaginal ultrasound (3D-TVUS), which allows the operator to place a high-frequency endocavitary ultrasound transducer in close proximity to target pelvic organs, thus improving image resolution. With the advent of high-resolution vaginal probes, 3D-TVUS has recently been adopted in the gynecological sciences. By enabling multiplanar displays, which simultaneously visualize the three orthogonal scan planes, 3D-TVUS boasts the additional advantage of being able to obtain anatomical views which are often unattainable by TAS or 2D-TVUS and also due to the fact that the coronal plane is easily accessible. The coronal views show the relationship between the endometrium and the myometrium at the uterine fundus, delineate the entire cervical canal and visualize the cornual angles. Intraoperative 2D-TVUS/3D-TVUS, however, would lengthen the duration of HA, potentially resulting in fluid overload. Instead, preoperative 3D-TVUS during the mid-menstrual phase would be more informative and accurate as it can be performed for a longer period with better image quality. Ultrasonographic data can be rapidly acquired preoperatively and stored for retrospective analysis with no loss of information. One can also "scroll" in realtime through the acquired volume that can be rotated and magnified. 3D-TVUS can give the surgeon preoperative detailed information about the cavity including which segments are obliterated, the extent of obliteration and the functional state of the endometrium. 3D-TVUS is 
considered as an optimal diagnostic test as it is non-invasive, safe, painless, widely available, inexpensive and applicable to all women regardless of their pretest probability of having a particular condition of interest. Most importantly, 3D-TVUS can explore the areas where a hysteroscope may have difficulties reaching, avoiding possible errors by the surgeon which could lead to failure to retrieve the fallopian tube ostia, create a false passage or even cause uterine perforation. To the best of our knowledge, there has been no published literature focusing on the use of preoperative 3D-TVUS in intraoperative judgement during HA in IUA patients. In this study, we used the data obtained from 3D-TVUS to make proper surgical planning, informed the patients about the current condition of their endometrial cavity and the potential course of their treatment, and capitalized on the information retrieved for intraoperative judgement while also minimizing risks and complications during HA.

\section{Methods}

\section{Patients}

This study was designed as a prospective non-randomized controlled study. Patients were collected from March, 2018 to December, 2018. After strict screening, 362 patients with IUA who met the inclusion criteria were enrolled. Of these, 182 patients received transvaginal 3D-TVUS examination during the secretory phase of their menstrual cycle before their operation (study group), and 180 patients did not receive transvaginal $3 \mathrm{D}-\mathrm{TVUS}$ examination before their operation (control group). All 3D-TVUS images were reconfirmed by the same senior and experienced doctor.

\section{Inclusion criteria}

(I) Aged 18-45 years, fertility seeking patients or patients with menstrual outflow obstruction.

(II) Diagnosed as IUA by diagnostic hysteroscopy with AFS score ranging from 5 to 12 .

(III) At least one unilateral fallopian tube ostium is not visible, confirmed by preoperative diagnostic hysteroscopy.

\section{Exclusion criteria}

(I) Cervical or endometrial lesions.

(II) Serious heart, liver or renal insufficiency.

(III) Patients with serious nervous system diseases, who are unable to take care of themselves in daily life or unable for undergo relevant treatment.
(IV) Surgical intolerance or inability to follow the doctor's advice for review or follow-up.

(V) Congenital malformation of uterus.

\section{D-TVUS examination}

GE VOLUSON E8 ultrasound instrument (GE Healthcare $\mathrm{GmbH} \&$ Co OG, Tiefenbach, Styria, Austria) with the two-dimensional volume probe in the cavity and the realtime three-dimensional volume probe were used for the preoperative three-dimensional ultrasound examination. Patients from the study group underwent preoperative transvaginal 3D-TVUS during the secretory phase of the menstrual cycle, using 7.5 MHz IC5-9D vaginal probe. During the examination, the patients emptied their bladder and were placed in the lithotomy position. Routine two-dimensional ultrasonographic examination was performed first. During 2D-ultrasound, the integrity of the endometrial layer was assessed to look for disruptions of the endometrial-myometrial junction. Adhesions on ultrasound are seen as bands of myometrial tissue traversing the endometrial cavity and adjoining the opposing uterine walls. After rotating the real-time three-dimensional volume probe, the panoramic technology was used to obtain the overall image information and select the target area. During the 3D-ultrasound examination, IUA are seen with the characteristic appearance of hyperechoic areas within the endometrium. Finally, the information was stored on a removable hard disk for further evaluation and calculation.

\section{Surgical procedure}

Hysteroscopic adhesiolysis (HA) was performed within 3-7 days following menstruation, with the patient placed in the lithotomy position and given intravenous anaesthesia. Patients fasted for 6-8 hours before surgery. A sterile saline solution was used to distend the uterus. Distension pressure was $110-120 \mathrm{mmHg}$ with a flow rate of $300-350 \mathrm{~mL} / \mathrm{min}$. The operation was monitored by transabdominal ultrasound (TAS). After routine disinfection and draping, hysteroscopy was carried out using an operative hysteroscope with an outer sheath diameter of $5.4 \mathrm{~mm}$ and a 5-Fr working channel (KARL STORZ SE \& Co. KG, Tuttlingen, Baden-Württemberg, Germany). The hysteroscope was introduced into the cervical canal through the cervix with the aim of reaching the intrauterine cavity. The adhesions located in the central part of the uterine cavity were usually dissected 


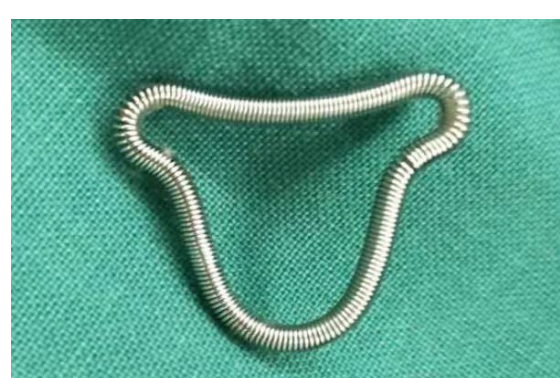

Figure 1 A uterine-shaped stainless-steel IUD. IUD, intrauterine device.

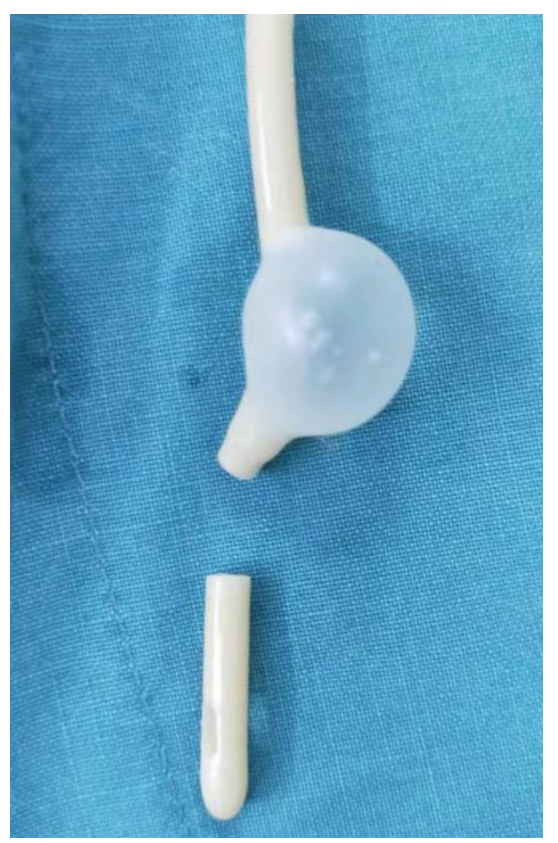

Figure 2 A double channel, 12-Fr Foley catheter balloon, with the top catheter portion beyond the balloon removed.

first and then the lateral adhesions were cut up. First, tubal ostia, which is the anatomical landmark during HA, needed to be retrieved with or without preoperative 3D-TVUS. Then, upon its visualization, the adhesions could be easily dissected. In cases of intrauterine anatomy distortion caused by adhesions, 5-Fr double action forceps were used under TAS monitoring, using a blunt spreading dissection technique (5) to separate the adhesions and anatomically reveal the uterine cavity. If the intrauterine anatomy was clear, the IUA were separated with 5-Fr single action sharp scissors and the scar tissue covering the intrauterine wall was treated with a "cold scissors ploughing technique" (6) until the entire uterine cavity had been opened successfully with clearly visible bilateral fallopian tube ostia. A uterine-shaped stainless-steel intrauterine device (IUD) (Figure 1) was then inserted into the uterine cavity, with its position checked via hysteroscopy to ensure that the size of the IUD matched the uterine cavity size and that the IUD was correctly positioned (7). A double channel, 12-Fr Foley catheter balloon, with the top catheter portion beyond the balloon removed (Figure 2), was inserted into the uterine cavity and distended using $2.5 \mathrm{~mL}$ of sterile saline with the balloon in the center of the uterine-shaped IUD. Three $\mathrm{mL}$ hyaluronic acid gel was then injected into the uterine cavity through the catheter. Postoperatively, the intrauterine balloon was kept in place for 7-14 days for patients with severe IUA and for 2-3 days for patients with mild and moderate IUA. For patients with only lower segment IUA, no IUD was used, and instead, a distended Foley catheter balloon was left in situ for up to 3 weeks.

\section{Postoperative follow up hysteroscopy recommendation:}

For patients with an AFS score $\geq 8$, a two-time followup strategy was implemented (the first follow-up is done one month after initial surgery, and the second one, 3 months after the first follow up hysteroscopy). For those with an AFS score $<8$, a one-time follow-up strategy was conducted (after 3 months of the initial surgery). During the last hysteroscopic follow-up procedure, hysteroscopic tubal catheterization and hydrotubation was performed to diagnose tubal patency following HA. Patients were followed up to one year after the last hysteroscopic intervention or until further improvement was likely to be impossible.

\section{Statistical analysis}

Statistical software SAS.9.4 was used to analyze the collected data. Binary logistic regression analysis and logistic regression analysis of unordered independent categorical variables were used in this study, and a Wilcoxon rank-sum test was used for variables that did not meet the requirements of a normality test. In addition, Pearson chisquare test or Fisher's Exact test was used to examine the association between categorical variables. A $\mathrm{P}$ value $<0.05$ was considered statistically significant and all statistical tests were two-tailed. 
Table 1 Analysis of variables between groups with unilaterally invisible tubal ostium

\begin{tabular}{|c|c|c|c|}
\hline Variables & Control group & Study group & $P$ value \\
\hline Age & & & 0.2674 \\
\hline Mean $\pm S D$ & $32.47 \pm 4.71$ & $31.60 \pm 4.86$ & \\
\hline Median [min, max] & $32.0[23,43]$ & $31.0[22,46]$ & \\
\hline Gravidity & & & 0.7429 \\
\hline Mean \pm SD & $3.38 \pm 1.75$ & $3.32 \pm 1.76$ & \\
\hline Median [min, max] & $3.0[0,10]$ & $3.0[0,11]$ & \\
\hline Parity & & & 0.7031 \\
\hline Mean \pm SD & $0.74 \pm 1.43$ & $0.58 \pm 0.81$ & \\
\hline Median [min, max] & $0.0[0,9]$ & $0.0[0,4]$ & \\
\hline Abortion & & & 0.6715 \\
\hline Mean \pm SD & $2.47 \pm 1.49$ & $2.47 \pm 1.74$ & \\
\hline Median [min, max] & $2.0[0,7]$ & $2.0[0,10]$ & \\
\hline Menstruation & & & 0.0851 \\
\hline Normal menses & $10(13.51 \%)$ & $7(9.33 \%)$ & \\
\hline Hypomenorrhea & $62(83.78 \%)$ & $60(80.00 \%)$ & \\
\hline Amenorrhea & $2(2.70 \%)$ & $8(10.67 \%)$ & \\
\hline Total & $74(100.00 \%)$ & $75(100.00 \%)$ & \\
\hline \multicolumn{2}{|c|}{ Preoperative cornual adhesions } & & $<0.0001$ \\
\hline No adhesion & $30(40.54 \%)$ & $9(12.00 \%)$ & \\
\hline Unilateral adhesions & $43(58.11 \%)$ & $65(86.67 \%)$ & \\
\hline Bilateral adhesions & $1(1.35 \%)$ & $1(1.33 \%)$ & \\
\hline Total & $74(100.00 \%)$ & $75(100.00 \%)$ & \\
\hline Preoperative AFS score & & & 0.0969 \\
\hline Mean \pm SD & $8.58 \pm 1.68$ & $9.12 \pm 1.59$ & \\
\hline Median [min, max] & $8.0[5,12]$ & $10.0[6,12]$ & \\
\hline Causes of IUA & & & 0.5939 \\
\hline Dilatation and curettage & $64(86.49 \%)$ & $67(89.33 \%)$ & \\
\hline $\begin{array}{l}\text { Other uterine cavity } \\
\text { operation }\end{array}$ & $10(13.51 \%)$ & $8(10.67 \%)$ & \\
\hline Total & 74 (100.00\%) & 75 (100.00\%) & \\
\hline
\end{tabular}

\section{Results}

\section{Surgical outcomes of unilaterally invisible tubal ostium}

Analysis of variables between groups with unilaterally invisible tubal ostium

The median $( \pm \mathrm{SD})$ ages of women at the time of adhesiolysis were $31.60 \pm 4.86$ and $32.47 \pm 4.71$ years for the study group and the control group, respectively $(\mathrm{P}=0.2674)$. Preoperative mean AFS scores were 9.12 \pm 1.59 and $8.58 \pm 1.68$ for the study group and the control group, respectively $(\mathrm{P}=0.0969)$. There were no statistically significant differences between the groups in terms of pregnancy history, menstruation and uterine cavity operations preoperatively $(\mathrm{P}>0.05)$, but for the study group, the cornual adhesions were more serious than those of the control group $(\mathrm{P}<0.0001)$ (details in Table 1).

\section{Multivariate logistic regression analysis of variables affecting retrieval rate of the unilaterally invisible fallopian tube ostium}

The variables with $\mathrm{P}<0.1$ in univariate analysis were put into the logistic regression analysis model, including menstruation, preoperative AFS score, 3D-TVUS, and preoperative cornual adhesions. Multivariate logistic regression analysis showed that: 3D-TVUS was the main factor that influenced the postoperative exposure rate of clearly visible tubal ostia ( $\mathrm{P}=0.0121, \mathrm{OR}=3.271,95 \% \mathrm{CI}$ : 1.296-8.251) (details in Table 2).

\section{Surgical outcomes of bilaterally invisible tubal ostia}

Analysis of variables between groups with bilaterally invisible tubal ostia

The median $( \pm$ SD) ages of women at the time of adhesiolysis were $32.27 \pm 5.02$ and $32.38 \pm 4.83$ years for the study group and the control group, respectively $(\mathrm{P}=0.8783)$. Preoperative mean AFS scores were $10.07 \pm 1.43$ and $10.02 \pm 1.59$ for the study group and the control group, respectively ( $\mathrm{P}=0.8962)$. There were no statistically significant differences between the groups in terms of pregnancy history, menstruation and preoperative uterine cavity surgeries $(\mathrm{P}>0.05)$, but the cornual adhesions of the study group were more serious than those of the control group ( $\mathrm{P}=0.0196)$ (details in Table 3).

Multivariate logistic regression analysis of variables affecting retrieval rate of the bilaterally invisible fallopian tube ostia

The variables with $\mathrm{P}<0.1$ in univariate analysis were put into the logistic regression analysis model of ordered classification, including both groups and preoperative cornual adhesions. Multivariate logistic regression analysis including preoperative: cornual adhesion and bilateral adhesions of tubal ostia showed that 3D-TVUS 
Table 2 Multivariate logistic regression analysis of variables affecting retrieval rate of unilaterally invisible fallopian tube ostium

\begin{tabular}{|c|c|c|c|c|c|c|}
\hline Effect & Degree of freedom & Estimate & Standard error & Wald Chi-square & $95 \% \mathrm{Cl}$ & $P$ value \\
\hline Menstruation & 2 & & & 1.2850 & & 0.5260 \\
\hline Preoperative AFS score & 1 & & & 3.6986 & & 0.0545 \\
\hline Group & 1 & & & 6.2979 & & 0.0121 \\
\hline \multicolumn{7}{|l|}{ Menstruation } \\
\hline Hypomenorrhea & 1 & 0.9046 & 0.8339 & 1.1768 & & 0.2780 \\
\hline Amenorrhea & 1 & 1.4542 & 1.3867 & 1.0997 & & 0.2943 \\
\hline Preoperative AFS score & 1 & -0.3528 & 0.1835 & 3.6986 & & 0.0545 \\
\hline \multicolumn{7}{|l|}{ Menstruation } \\
\hline Hypomenorrhea vs. normal menses & & 2.471 & & & $0.482-12.666$ & \\
\hline Amenorrhea vs. normal menses & & 4.281 & & & $0.283-64.845$ & \\
\hline Preoperative AFS score & & 0.703 & & & $0.490-1.007$ & \\
\hline Group: study group vs. control group & & 3.271 & & & $1.296-8.251$ & \\
\hline
\end{tabular}

and preoperative cornual adhesions were the main factors that affect postoperative exposure rate of clearly visible tubal ostia (group: $\mathrm{P}<0.0001$, OR $=84.638,95 \%$ CI: 26.940-265.905; preoperative unilateral cornual adhesion: $\mathrm{P}=0.0006, \mathrm{OR}=15.372$, 95\% CI: 3.228-73.189; preoperative bilateral cornual adhesions: $\mathrm{P}<0.0001, \mathrm{OR}$ =46.849, 95\% CI: 13.240-165.778) (details in Table 4).

\section{Postoperative retrieval rate of fallopian tube ostia and AFS score reduction}

Postoperatively, mean AFS scores were 4.97 \pm 2.53 and $6.35 \pm 2.89$ for the study group and the control group, respectively. AFS reduction in the study group was better than in the control group; $4.71 \pm 2.05$ and $3.05 \pm 2.21$ respectively with a significant $\mathrm{P}$ value of $<0.0001$. There were 149 patients (74 in control group and 75 in study group) with unilateral invisible fallopian tube ostium preoperatively and 213 patients (106 in control group and 107 in study group) with bilateral invisible fallopian tube ostia preoperatively. For the study group, with the help of preoperative 3D-TVUS the entire uterine cavity has been more significantly and successfully opened with more clearly visible fallopian tube ostia: (details in Table 5). Rank variance analysis showed that preoperative transvaginal 3-D ultrasound was the most important factor for AFS score reduction (details in Tables 6,7).

\section{Discussion}

HA is considered as the criterion standard in the management of IUA as it enables the lysis of adhesions under direct vision and with magnification (8). The primary objective of this intervention is to restore the normal volume, shape of the intrauterine cavity and cervical canal, and to access and visualize the fallopian tubes ostia, which are the anatomical landmarks during HA. Difficulties arise in cases of moderate or severe IUA, when unilateral or bilateral ostia cannot be seen during diagnostic hysteroscopy or in cases of complete/ extensive obliteration of the uterine cavity and cervical canal stenosis when the hysteroscope cannot be inserted beyond the point of obliteration. Under such circumstances, the intrauterine anatomy is usually distorted and the anatomical landmarks are not recognizable, posing a significant challenge for the hysteroscopist. As these conditions can cause difficulties in intraoperative decision-making, any 
Table 3 Analysis of variables between groups with bilaterally invisible tubal ostia

\begin{tabular}{|c|c|c|c|}
\hline Variables & Control group & Study group & $P$ value \\
\hline Age & & & 0.8783 \\
\hline Mean \pm SD & $32.38 \pm 4.83$ & $32.27 \pm 5.02$ & \\
\hline Median [min, max] & $32.5[23,43]$ & $33.0[20,42]$ & \\
\hline Gravidity & & & 0.6702 \\
\hline Mean \pm SD & $3.40 \pm 1.84$ & $3.46 \pm 2.34$ & \\
\hline Median [min, max] & $3.0[1,10]$ & $3.0[0,12]$ & \\
\hline Parity & & & 0.4534 \\
\hline Mean \pm SD & $0.63 \pm 0.69$ & $0.72 \pm 0.79$ & \\
\hline Median [min, max] & $1.0[0,4]$ & $1.0[0,5]$ & \\
\hline Abortion & & & 0.5179 \\
\hline Mean \pm SD & $2.57 \pm 1.72$ & $2.53 \pm 2.04$ & \\
\hline Median [min, max] & $2.0[0,9]$ & $2.0[0,11]$ & \\
\hline Menstruation & & & 0.5164 \\
\hline Normal menses & $0(0.00 \%)$ & $4(3.74 \%)$ & \\
\hline Hypomenorrhea & $79(74.53 \%)$ & $77(71.96 \%)$ & \\
\hline Amenorrhea & $27(25.47 \%)$ & $26(24.30 \%)$ & \\
\hline Total & \multicolumn{2}{|c|}{$106(100.00 \%) 107$ (100.00\%) } & \\
\hline \multicolumn{2}{|c|}{ Preoperative cornual adhesions } & & 0.0196 \\
\hline No adhesion & $25(23.58 \%)$ & $10(9.35 \%)$ & \\
\hline Unilateral adhesions & $6(5.66 \%)$ & $7(6.54 \%)$ & \\
\hline Bilateral adhesions & $75(70.75 \%)$ & $90(84.11 \%)$ & \\
\hline Total & \multicolumn{2}{|c|}{106 (100.00\%) 107 (100.00\%) } & \\
\hline Preoperative AFS score & & & 0.8962 \\
\hline Mean \pm SD & $10.02 \pm 1.59$ & $10.07 \pm 1.43$ & \\
\hline Median [min, max] & $10.0[5,12]$ & $10.0[6,12]$ & \\
\hline Causes of IUA & & & 0.8150 \\
\hline Dilatation and curettage & $90(84.91 \%)$ & $88(82.24 \%)$ & \\
\hline $\begin{array}{l}\text { Other uterine cavity } \\
\text { operation }\end{array}$ & $16(15.09 \%)$ & 19 (17.76\%) & \\
\hline Total & $106(100.00 \%)$ & 107 (100.00\%) & \\
\hline
\end{tabular}

IUA, intrauterine adhesion.

attempt of entry might lead to failure to retrieve the cavity and ostia, creation of false passages, uterine perforation and entail several other risks.

Several techniques have been proposed to lyse adhesions. Coccia et al. in 2001 reported that IUA patients can be treated by Pressure Lavage under Ultrasound Guidance (PLUG) method using the distention medium used for hysteroscopy for adhesiolysis (9). The Seldinger technique (10) under ultrasound (US) guidance followed by balloon-aided cervical dilation has also been proposed for treatment of IUA. But both of the aforesaid techniques are useful only for treatment of mild adhesions and have low efficiency in treatment of moderate to severe adhesions. For moderate to severe IUA, the myometrial scoring method was proposed, in which a series of 6-8 incisions are made on the myometrium, from the fundus to the isthmus to a depth of $4 \mathrm{~mm}$ using a Collins knife electrode (11). However, as electrosurgical energy poses a risk of endometrial destruction and there is a tendency for IUA to reoccur, its use is not recommended for fertility-seeking patients (6). Roy et al., in 2010, reported on 89 infertile patients with IUA who underwent HA with concomitant laparoscopy (12). They stated that concurrent laparoscopy is helpful for confirming tubal patency and ruling out other pelvic pathologies to elucidate the boundaries of adhesiolysis by observing the transillumination. However, they encountered 2 cases of uterine perforation during the procedure. Thomson et al., in 2007 reported on 30 patients with IUA who underwent HA under fluoroscopic guidance, which allows the surgeon to view islands of endometrium behind scar tissue in an obliterated uterine cavity. The use of a Tuohy needle is used in parallel to hysteroscopy $(13,14)$ through which a radiopaque dye is injected into an area of dense scar at the point where the cavity is obliterated. Any pockets of endometrium beyond the adhesive area can then be identified using fluoroscopy and this area can be opened up by sharp dissection under hysteroscopic view (13). However, fluoroscopy can result in relatively high doses of radiation, especially for complex interventional procedures which require its administration for a longer period of time. Another limitation of this technique is that it involves the use of an image intensifier and requires the presence of a radiographer throughout the HA procedure. Studies have shown that HA under laparoscopic or fluoroscopic guidance is not necessary to reduce the risk of uterine perforation (15). Rather, the use of TAS during HA is a preferred and current option as it is non-invasive. TAS has been described as a technique to guide hysteroscopic division of IUA $(16,17)$ and the availability and familiarity of sonography to gynecologists makes this option easy to implement. In our study, concomitant TAS was performed on every patient intraoperatively to guide the extent of adhesiolysis.

However, TAS has its limitations; the 2D imaging quality of portable TAS is not very accurate or informative during the proliferative phase of the menstrual cycle; during which the endometrium is very thin. Moreover, 
Table 4 Multivariate logistic regression analysis of variables affecting retrieval rate of the bilaterally invisible fallopian tube ostia

\begin{tabular}{|c|c|c|c|c|c|c|}
\hline Effect & Degree of freedom & Estimate & Standard error & Wald Chi-square & $95 \% \mathrm{Cl}$ & $P$ value \\
\hline \multicolumn{7}{|l|}{ Type 3 effect analysis } \\
\hline Group & 1 & & & 57.7457 & & $<0.0001$ \\
\hline Preoperative cornual adhesions & 2 & & & 35.5981 & & $<0.0001$ \\
\hline \multicolumn{7}{|l|}{ Maximum Likelihood estimate } \\
\hline \multicolumn{7}{|l|}{ Preoperative cornual adhesions } \\
\hline Unilateral adhesions & 1 & 2.7325 & 0.7962 & 11.7783 & & 0.0006 \\
\hline Bilateral adhesions & 1 & 3.8469 & 0.6448 & 35.5981 & & $<0.0001$ \\
\hline \multicolumn{7}{|l|}{ Odds ratio estimate } \\
\hline Unilateral adhesions vs. no adhesion & & 15.372 & & & $3.228-73.189$ & \\
\hline Bilateral adhesions vs. no adhesion & & 46.849 & & & $13.240-165.778$ & \\
\hline
\end{tabular}

Table 5 Postoperative retrieval rate of fallopian tube ostia and AFS score reduction

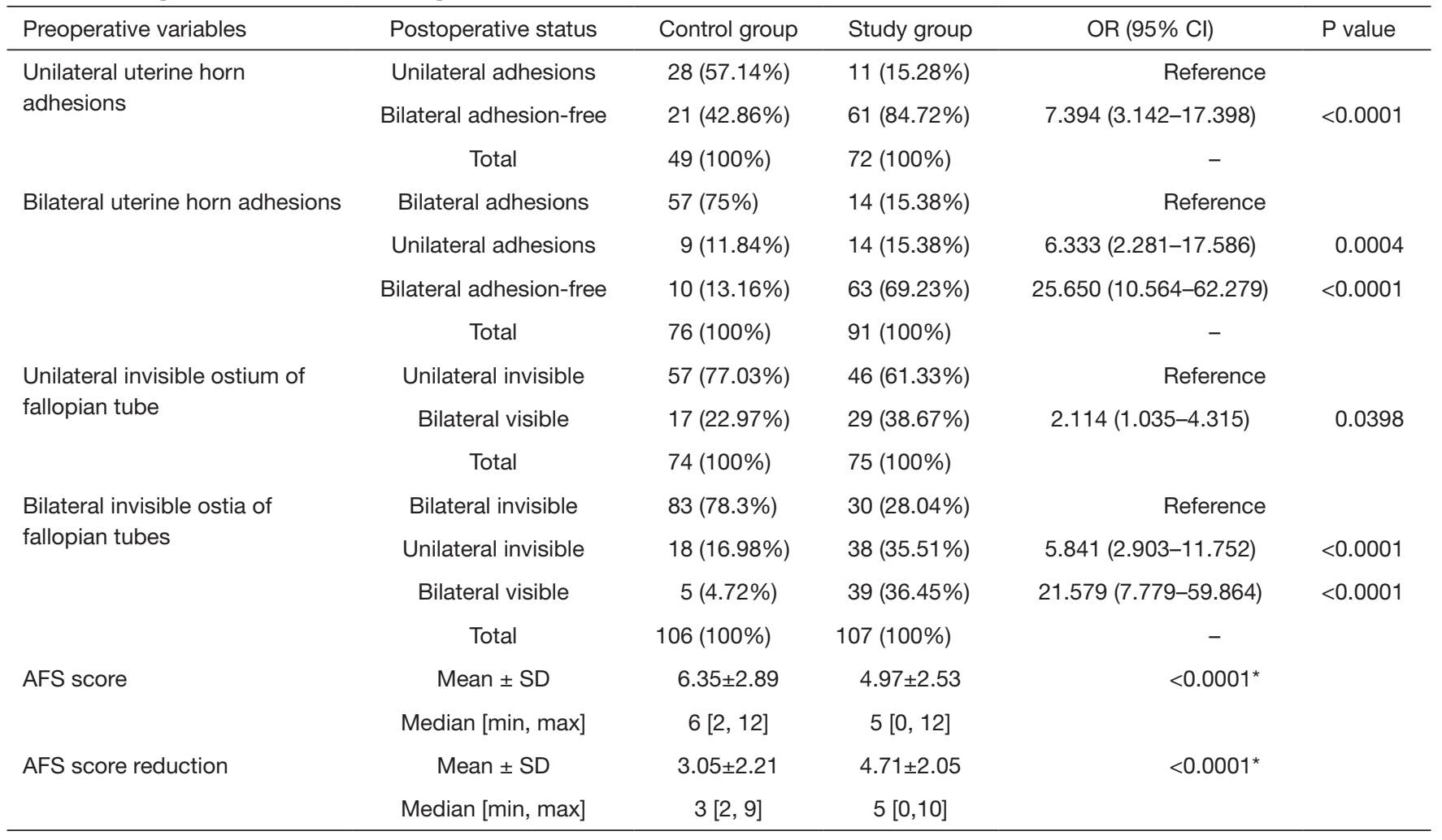


Table 6 Rank variance analysis of AFS score reduction (part I)

\begin{tabular}{lccc}
\hline Variables & Control group & Study group & P value \\
\hline Preoperative AFS score & & & 0.0969 \\
Mean \pm SD & $8.58 \pm 1.68$ & $9.12 \pm 1.59$ & \\
Median [min, max] & $8.0[5,12]$ & $10.0[6,12]$ & \\
Postoperative AFS & & & $<0.0001^{*}$ \\
score & & & \\
Mean \pm SD & $6.35 \pm 2.89$ & $4.97 \pm 2.53$ & \\
Median [min, max] & $6[2,12]$ & $5[0,12]$ & \\
AFS score reduction & & & $<0.0001^{*}$ \\
Mean \pm SD & $3.05 \pm 2.21$ & $4.71 \pm 2.05$ & \\
Median [min, max] & $3[-2,9]$ & $5[0,10]$ & \\
\hline
\end{tabular}

Table 7 Rank variance analysis of AFS score reduction (part II)

\begin{tabular}{cccc}
\hline Effect & Degree of freedom & Estimate & P value \\
\hline Group & 1 & 43.2971 & $<0.0001$ \\
\hline
\end{tabular}

uterine perforation was reported in as many as $5 \%$ of cases (17-19) when TAS was used as the sole adjunctive aid during HA. This led us to adopt a different non-invasive approach; through preoperative 3D-TVUS as it provided detailed information about the location, extent and degree of adhesions within the uterine cavity. Depending on the location of the adhesions, surgical decisions can be made preoperatively and implemented intraoperatively. From the data obtained from 3D-TVUS, the surgeon anticipates what to see and where to anticipate adhesions. The absence of an expected finding in a specific area or segment might indicate that the hysteroscope is not in the proper layer or location. In our study, a case of previously failed HA, where the left cornual cavity could not be identified, was sent for a 3D-TVUS, which demonstrated the cornual cavity clearly. We estimated that most probably the direction of the surgery was wrong initially or the surgeon did not reach the proper depth of the anatomy. For the repeat surgery, data from 3D-TVUS was interpreted preoperatively and was used intraoperatively to successfully
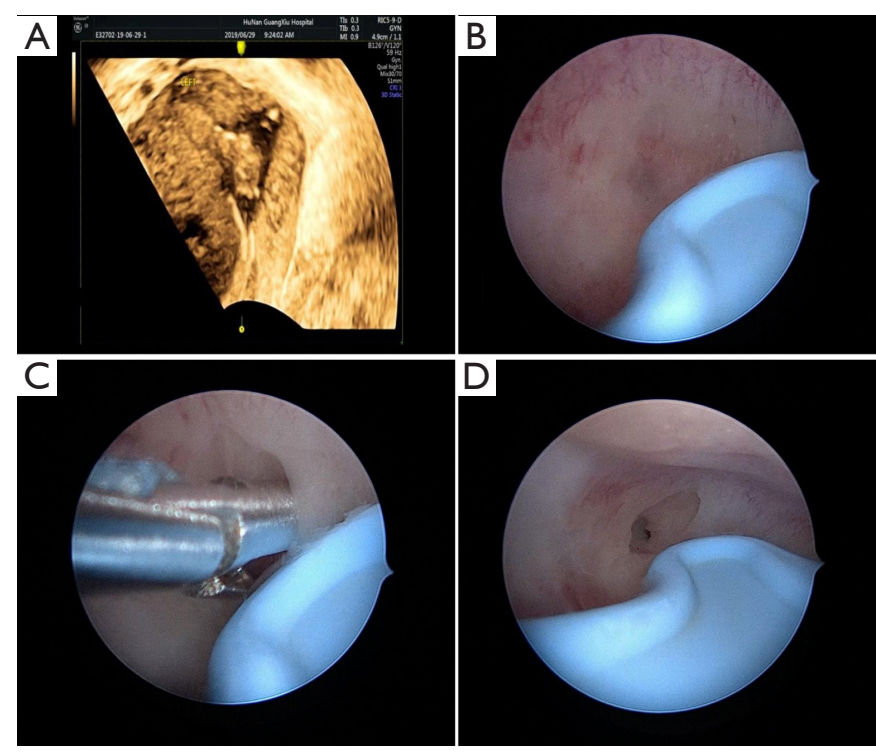

Figure 3 Data from 3D-TVUS was interpreted preoperatively and was used intraoperatively to successfully reveal the left cornual cavity. (A) The image of 3D-TVUS showed the left cornu was visible but its location was deeper than normal, and the right cornu and tubal ostium were faintly visible; (B) the left cornu and tubal ostium were not visible during hysteroscopy; (C) with the help of preoperative 3D-TVUS, our hysteroscopist separated adhesions at the left cornu; (D) a clearly visible tubal ostium was exposed. 3D-TVUS, three-dimensional transvaginal ultrasound. 

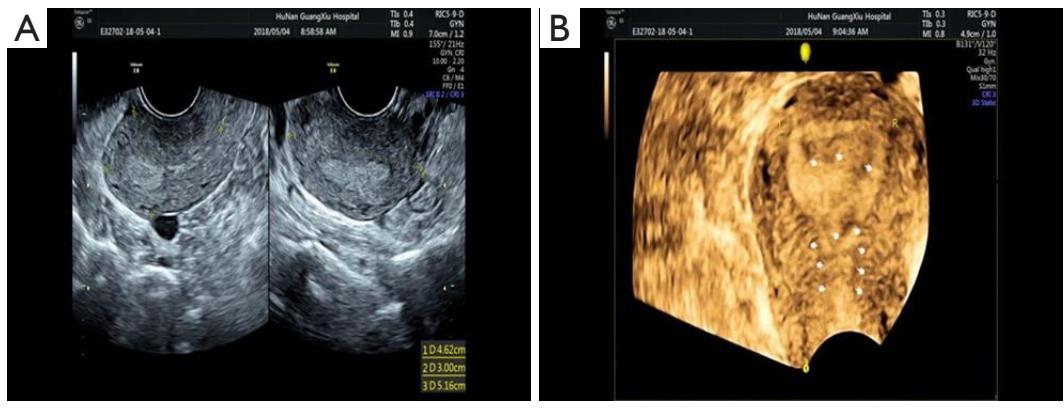

Figure 4 3D-TVUS guides and informs the operator about the condition of the endometrium and the uterine cavity beyond the point of obliteration. (A) 2D-TVUS showing a normal sized uterus with normal contour, a smooth surface and uneven endometrial thickness; (B) 3D-TVUS showing that the lower part of the uterine cavity is closed, the middle section is narrowed, and the endometrium is continuously interrupted. However, both cornual angles are clear. 3D-TVUS, three-dimensional transvaginal ultrasound.

reveal the left cornual cavity (Figure $3 A, B, C, D$ ). Apart from the size and contour of the uterus, $3 \mathrm{D}$-TVUS is indicative of the distance between the uterine isthmus and fundus, the intercornual distance, the presence or absence of healthy endometrium in different segments of the uterine cavity. Hysteroscopists are often blinded by severe lower segment adhesions/obliteration and not only face difficulties in retrieving tubal ostia or reaching the uterine cavity but are also unaware what to expect in the middle and upper segments. 3D-TVUS guides and informs the operator about the condition of the endometrium and the uterine cavity beyond the point of obliteration and can also indicate the distance between the point of obliteration and ostium (Figure 4A,B). 3D-TVUS can analyze and explore areas where the hysteroscope cannot reach. It can not only demonstrate the angular cavity clearly but can also inform the hysteroscopist about its condition. From time to time, in cases of lower segment uterine cavity obliteration or severe IUA, a false passage may be created. When the latter is diagnosed preoperatively with the aid of 3D-TVUS, the hysteroscopist knows exactly where to expect the false passage and necessary precautions can be taken intraoperatively so as to recognize it and prevent perforation.

In our study, the study group consisted of patients with higher mean AFS scores than those in the control group, yet we noted that, postoperatively, their mean AFS score and mean AFS score reduction were better; $4.97 \pm 2.53$ and $4.71 \pm 2.05$, respectively, with $P$ value $<0.0001$ in both instances. The retrieval and visibility of fallopian tube ostia were more significant in the study group. No cases of uterine perforation were noted during HA in our study. As our findings are encouraging, we would recommend for all patients to undergo routine 3D-TVUS examinations prior to HA and a diagnostic hysteroscopy would less likely be needed.

\section{Conclusions}

Preoperative $3 \mathrm{D}$-TVUS has proved to have a very important role in intraoperative judgement and also in improving HA success rates.

\section{Acknowledgments}

Funding: Natural Science Foundation of China (Grant No. 81671492), the Hunan Science and Technology Department (Grant No. 2018SK2102).

\section{Footnote}

Conflicts of Interest: The authors have no conflicts of interest to declare.

Ethical Statement: The authors are accountable for all aspects of the work in ensuring that questions related to the accuracy or integrity of any part of the work are appropriately investigated and resolved. The study was approved by the Institutional Review Board (IRB) of Third Xiangya Hospital and Xiangya Hospital, Central South University (No. 2019-S455).

\section{References}

1. Diamond MP, Freeman ML. Clinical implications of postsurgical adhesions. Hum Reprod Update 
2001;7:567-76.

2. Hellebrekers BW, Trimbos-Kemper TC, Trimbos $\mathrm{JB}$, et al. Use of fibrinolytic agents in the prevention of postoperative adhesion formation. Fertil Steril 2000;74:203-12.

3. Yang JH, Chen MJ, Chen CD, et al. Optimal waiting period for subsequent fertility treatment after various hysteroscopic surgeries. Fertil Steril 2013;99:2092-6.e3.

4. Magos A. Hysteroscopic treatment of Asherman's syndrome. Reprod BioMed Online 2002;4:46-51.

5. Huang H, Cheng C, Johnson G, et al. Hysteroscopic Intrauterine Adhesiolysis Using a Blunt Spreading Dissection Technique With Double-action Forceps. Journal of Minimally Invasive Gynecology 2018;25:583-4.

6. Simsir C, Var T, Kalem MN, et al. Hysteroscopic treatment of Asherman's Syndrome. Cumhuriyet Medical Journal 2019;41:443-9.

7. Salma U, Xue M, Sayed AS, et al. Efficacy of Intrauterine Device in the Treatment of Intrauterine Adhesions. Biomed Res Int 2014;2014:589296.

8. Berman JM. Intrauterine adhesions. Semin Reprod Med 2008;26:349-55.

9. Coccia ME, Becattini C, Bracco GL, et al. Pressure Lavage under ultrasound guidance: A new approach for outpatient treatment of intrauterine. Fertil Steril 2001;75:601-6.

10. Seldinger SI. Catheter replacement of the needle in percutaneous arteriography: a new technique. Acta Radiol
Suppl (Stockholm) 2008;434:47-52.

11. Protopapas A, Shushan A, Magos A. Myometrial Scoring: A new technique for the management of severe Asherman's syndrome. Fertil Steril 1998;69:860-4.

12. Roy KK. Reproductive outcome following hysteroscopic adhesiolysis in patients with infertility due to Asherman's syndrome. Arch Gynecol Obstet 2010;281:355-61.

13. Broome JD, Vancaillie TG. Fluoroscopically guided hysteroscopic division of adhesions in severe Asherman syndrome. Obstet Gynecol 1999;93:1041-3.

14. Duffy S, Reid P, Sharp F. In-vivo studies of uterine electrosurgery. Br J Obstet Gynaecol 1992;99:579-82.

15. Fedele L, Bianchi S, Frontino G. Septums and synechiae: approaches to surgical correction. Clin Obstet Gynecol 2006;49:767-88.

16. Yu D, Wong YM, Cheong Y, et al. Asherman syndrome: one century later. Fertil Steril 2008;89:759-79.

17. Protopapas A, Shushan A, Magos A. Myometrial scoring: a new technique for the management of severe Asherman's syndrome. Fertil Steril 1998;69:860-4.

18. Zikopoulos KA. Live delivery rates in subfertile women with Asherman's syndrome after hysteroscopic adhesiolysis using the resectoscope or the Versapoint system. Reprod Biomed Online 2004;8:720-5.

19. McComb PF, Wagner BL. Simplified therapy for Asherman's syndrome. Fertil Steril 1997;68:1047-50.
Cite this article as: Burjoo A, Zhao X, Zou L, Liu X, Lei L, Zhang B, Xu D. The role of preoperative 3D-ultrasound in intraoperative judgement for hysteroscopic adhesiolysis. Ann Transl Med 2020;8(4):55. doi: 10.21037/atm.2020.01.06 